\title{
STATISTICAL METHODS IN THE IDENTIFICATION AND PREDICTION OF THE SOLAR X-RAY SPECTRAL LINES
}

\author{
SHAHINAZ YOUSEF
}

Astronomy Department, Faculty of Science, Cairo University, Cairo, Egypt

Summary. With the accumulation of numerous identifications of the solar X and XUV spectral lines, the need is felt for systemizing such identification. The results are presented below.

The degree of ionization distribution of spectral lines at different wavelength bands is shown in Figure 1, where each stroke represents a spectral line that has been identified in the solar spectrum. A preliminary list has been used in preparation of this figure.

It is obvious that the $\mathrm{X}$-ray spectral lines with wavelength below $60 \AA$ form two main sequences. The first one contains lines of light elements from carbon to sulphur. It starts from the XVI degree of ionization at $\lambda \geqslant 2 \AA$ and proceeds to lower stages of ionizations at longer wavelengths where it ends at the vth stage for $\lambda$ about $40 \AA$.

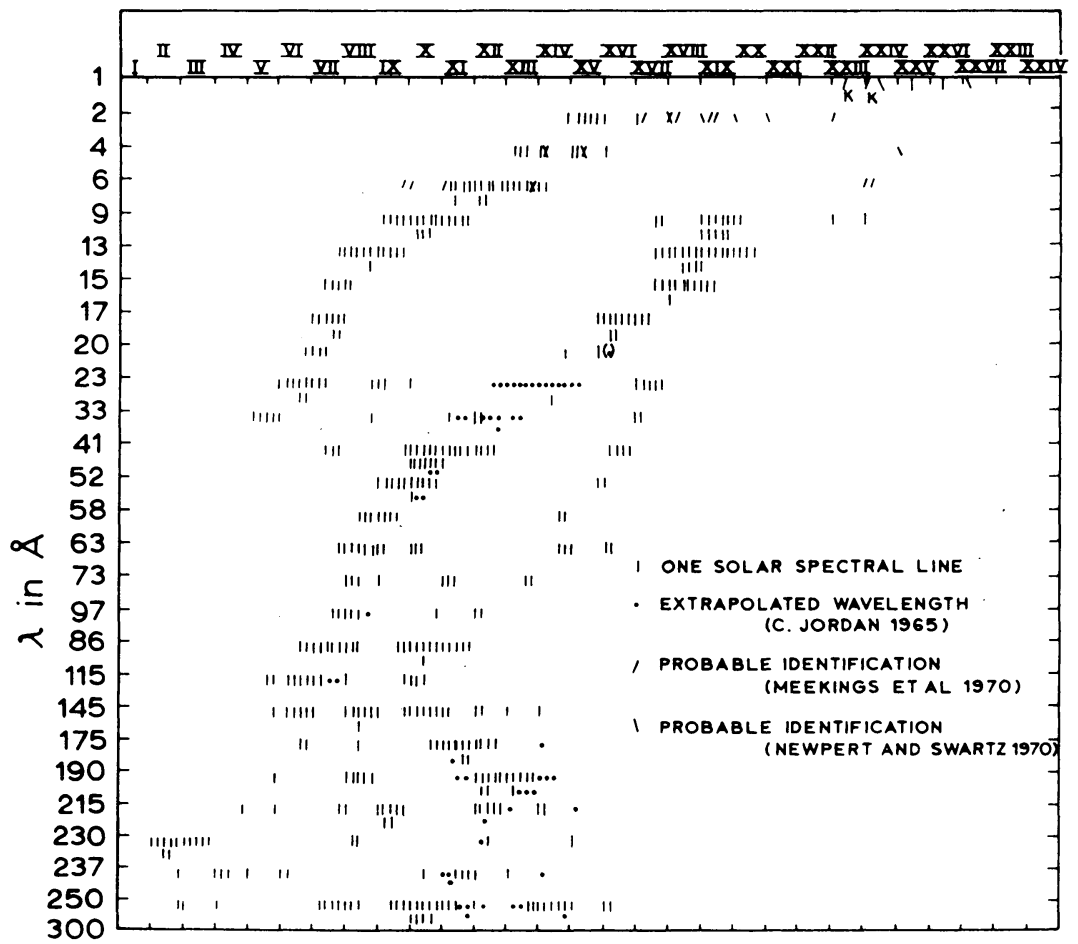

Fig. 1. The degree of ionization distribution of spectral lines at different wavelength bands. Each stroke represents a spectral line that has been identified in the solar spectrum. 
The second main sequence contains lines of heavier ions and is expected by extrapolation to start from the XXIII or XXIV degree of ionization for $4<\lambda<2 \AA$ and extends to the IX degree of ionization at $\lambda \leqslant 63 \AA$.

There is a gap of lines in the observed solar spectrum in this sequence between 20-40 $\AA$. However, a number of lines that would fit nicely in this gap was theoretically predicted by Jordan in 1965. It is thus suggested that more lines are expected to be observed in the solar spectrum in order to fill this gap. This expectation has been confirmed by Freeman and Jones (1970), who identified one line of S XII at $36.39 \AA$ and reported three other unidentified lines in the vicinity of $35 \AA$. Forecast is also made for the identification of more spectral lines in the XIX to XXV stages of ionization and wavelength between $2-9 \AA$ is made. This was partially confirmed by the identification of two Fe XXIII lines at $8.56 \AA$ and $8.8 \AA$ and a Fe XXII line at $8.98 \AA$ (Doschek et al., 1973).

In addition to these two main sequences, there might be an iron lines subsequent between 23 and $60 \AA$.

It is also noticed that some other lines lie off the sequence; these, in my opinion, are probably wrong and need re-identification. For instance, the lines at $\sim 11.0 \AA$ and $12 \AA$ identified by Chubb et al. (1964) as due to transitions in Fe XXIV and XXIII respectively are wrong. The first one was later re-identified by Connerade et al. (1970) in laboratory spectrum as due to Fe XIX. This identification would then make the line fit nicely in the sequence.

On plotting the distribution of ionization potential with wavelength for the same lines shown in Figure 1, all lines form one sequence only.

\section{References}

Chubb, T. H., Friedman, H., Kreplin, R. W.: 1964, Space Res. IV, 759.

Connerade, J. P., Peacock, N. J., and Speer, R. J.: 1970, Solar Phys. 14, 159.

Doschek, G. A., Meekins, J. F., and Cowan, Robert D.: 1973, Solar Phys. 29, 125.

Freeman, F. F. and Jones, F. B.: 1970, Solar Phys. 15, 288.

Jordan, Carole: 1965, Ph.D. Thesis, University of London.

Meekins, J. F., Dorschek, G. A., Friedman, H., Chubb, T. A., and Kreplin, R. W.: 1970, Solar Phys. 13, 198,

Neupert, W. and Swartz, M.: 1970, Astrophys. J. 160, L189. 